\title{
Experimental study: OXSEALIFE
}

Lara Oller ${ }^{1}$, Carlota Largo ${ }^{1}$, Iván Huercio ${ }^{1}$, Vanesa Escribano ${ }^{1}$, Aryeh Shander ${ }^{2}$

${ }^{1}$ Cirugía experimental. Hospital Universitario La Paz. IDIPAZ. Madrid. Spain.

${ }^{2}$ Englewood Hospital and Medical Center, New Jersey. Mount Sinai School of Medicine. NYC. USA.

\section{Introduction:}

Oxygen transportation in blood relies mostly on hemoglobin concentration, and the oxygen dissolved in plasma has a relatively minor contribution. This is due to the low rate of solubility of oxygen in blood plasma under physiologic condition and at atmospheric oxygen pressure. Hence, transfusion guidelines generally consider the hemoglobin concentration (alongside with the clinical status of the patient) but not the oxygen partial pressure. There are previous studies looking at the variation in the coefficient of solubility with temperature variations, but none alludes to the possible variation of oxygen solubility coefficient of blood plasma with fluid replacement.

\section{Purpose:}

To demonstrate that coefficient of solubility of oxygen in blood is not static but dynamic. It is generally assumed that the coefficient of solubility of oxygen in plasma is $0.0031 \mathrm{~mL} \mathrm{02/mmHg} \mathrm{P02/dl} \mathrm{plasma} \mathrm{but} \mathrm{we} \mathrm{hypothesize} \mathrm{that} \mathrm{this} \mathrm{value}$ might change as we do haemodynamic resuscitation following blood loss.

\section{Procedures:}

We measure dissolved oxygen and solubility coefficient in whole blood of swine and after dilution with Plasmalyte (P) and Hydroxyethyl starch HES 130/0.4 (V). Dilution is performed maintaining a mathematical ratio: $9 \mathrm{ml}$ of blood and $1 \mathrm{ml}$ of balanced solution, $8 \mathrm{ml}$ of blood and $2 \mathrm{ml}$ of balanced solution and so on. We measure hemoglobin concentration after each dilution with gasometer.

Then we centrifuge each sample and separate plasma from erythrocytes and platelets with a pipette. With an oxygen meter we measure dissolved oxygen in plasma $(\mathrm{mg} / \mathrm{L})$ avoiding the presence of bubbles at constant temperature $\left(36^{\circ} \mathrm{C}\right)$ and atmospheric pressure $(700 \mathrm{mmHg})$. Estimated p02 is $147 \mathrm{mmHg}(700 \mathrm{mmHg} \times 0,21)$ for all dilutions since atmospheric pressure is $700 \mathrm{mmHg}$ and atmospheric PO2 is 0,21 . After calculation of dissolved oxygen we calculate oxygen solubility coefficient by using this formula: Coefficient of solubility $(02 \mathrm{ml} / \mathrm{mm} \mathrm{Hg} / \mathrm{dl}$ plasma $)=$ DO plasma $/ \mathrm{pO2} / \mathrm{dl}$ plasma .

\section{Results:}

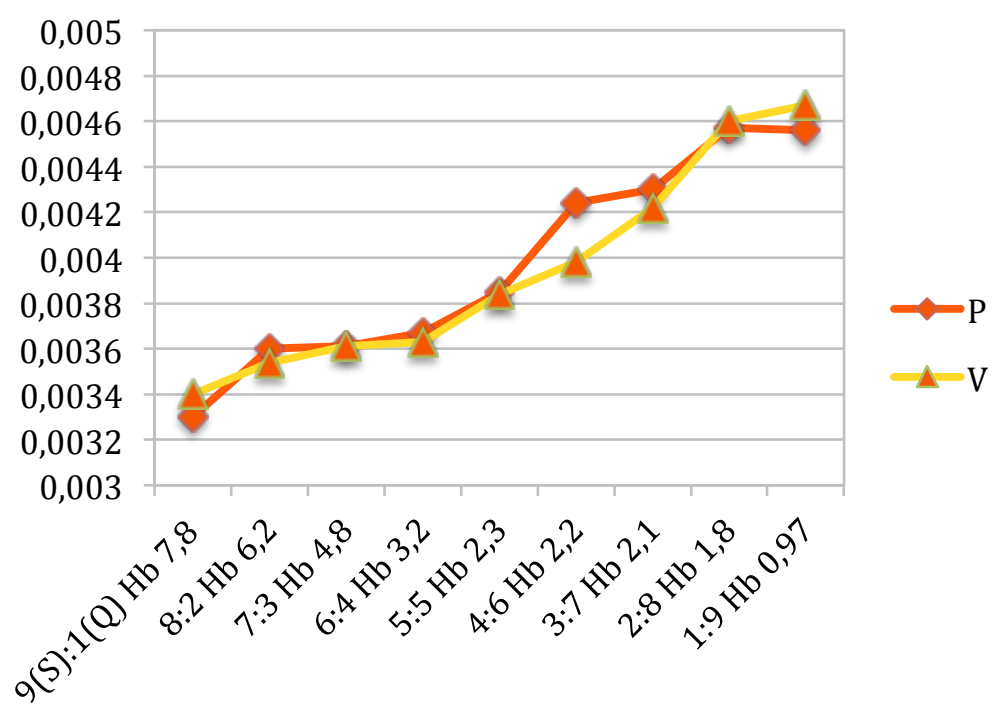

\section{Discussion:}

In face of severe anemia, the contribution made by oxygen dissolved in plasma can be essential to life. Modification of solubility coefficient of oxygen in blood plasma during haemodynamic resuscitation offers ways to augment this contribution. Now we know that as hemoglobin descends the contribution made by dissolved oxygen increases and can be as high as $35 \%$ of the total oxygen delivery. These new findings bring us to conclude that arterial oxygen content formula has to be reviewed. Now some questions arise. Since we know that solubility varies with pressure, temperature and salinity, why does this variation occur at constant temperature, pressure and salinity?

\section{Conclusions:}

Coefficient of solubility is not static but dynamic. More studies are needed to explore the oxygen solubility question(s). 